\title{
17. ESTIMATION OF ATTACHMENT RATE FROM THE DIFFERENT ECHO DURATION OF NIGHT- AND

\author{
DAY-TIME METEORS
}

\author{
P. GLÖDE \\ (Observatory for Ionospheric Research, Kühlungsborn, German Democratic Republic)
}

\begin{abstract}
The probability distributions of echo durations as measured on a $33 \mathrm{Mc} / \mathrm{sec}$ horizontally beamed radar at Kühlungsborn, G.D.R. $\left(54^{\circ} 07^{\prime} \mathrm{N}, 11^{\circ} 46^{\prime} \mathrm{E}\right)$ indicate significant differences between echo durations during night- and day-time. These differences are attributed to attachment during night, whereas the strong radiation detachment of electrons during day-time leaves effectively no negative ions. The nocturnal attachment rate found from the difference in the probability distributions is higher than the rates deduced from the aeronomical processes hitherto known in the meteor height range. The probable attachment processes, the rate coefficients and particle densities are listed as far as known.
\end{abstract}

\section{Introduction}

The duration of 'overdense' meteor echoes with line densities exceeding $10^{14}$ electrons/meter depends on the time through which a negative dielectric constant core exists. Besides the ambipolar diffusion, which leads to a gaussian diffusion distribution of electrons without changing the number of free electrons in the trail, the loss of free electrons by attachment to neutral atoms or molecules terminates the existence of such a reflecting core. Compared to the electrons, ions are ineffective for radio reflection in consequence of their heavier weight. Along with attachment, recombination has been considered, but as Kaiser (1953) and also McKinley (1961) demonstrated, it cannot seriously influence the echo duration. Upper-atmosphere turbulence could hasten the distortion of the dense core. Generally, attachment is considered to have the most important influence on echo duration. The influence of attachment was calculated by Davis et al. (1959) and Greenhow and Hall (1962). A general mathematical theory of attachment was presented by Manning (1964). Attachment demands our attention from two points of view:

(a) From the duration of the radio echo the mass of the meteor can be calculated, if besides the time constant of diffusion all other trail-disturbing processes, perhaps mainly the attachment process and its time dependence, are known.

(b) The attachment process itself is interesting for upper-atmosphere physics and plays an important role in the theory of the ionosphere, so that meteor observations could give experimental material for the aeronomical investigations. 
Attachment processes and their rates, considered with regard to meteors, have generally to be in agreement with aeronomical data found otherwise for the certain height range.

\section{Experimental Results}

With a $33 \mathrm{Mc} / \mathrm{sec}-10 \mathrm{~kW}$ pulse radar (pulse repetition frequency $25 \mathrm{c} / \mathrm{sec}$ ) at Kühlungsborn $\left(54^{\circ} 07^{\prime} \mathrm{N}, 11^{\circ} 46^{\prime} \mathrm{E}\right)$ normally used for radio aurora observation, meteor echoes have been recorded. The horizontally directed 4-element-Yagi antenna with a beam width of $60^{\circ}$ was fixed in a Northerly direction. The brightness-modulated display was recorded on a film moving continuously, so that the duration of meteor echoes exceeding $0.5 \mathrm{sec}$ was measurable (Figure 1 ). Due to the horizontally directed

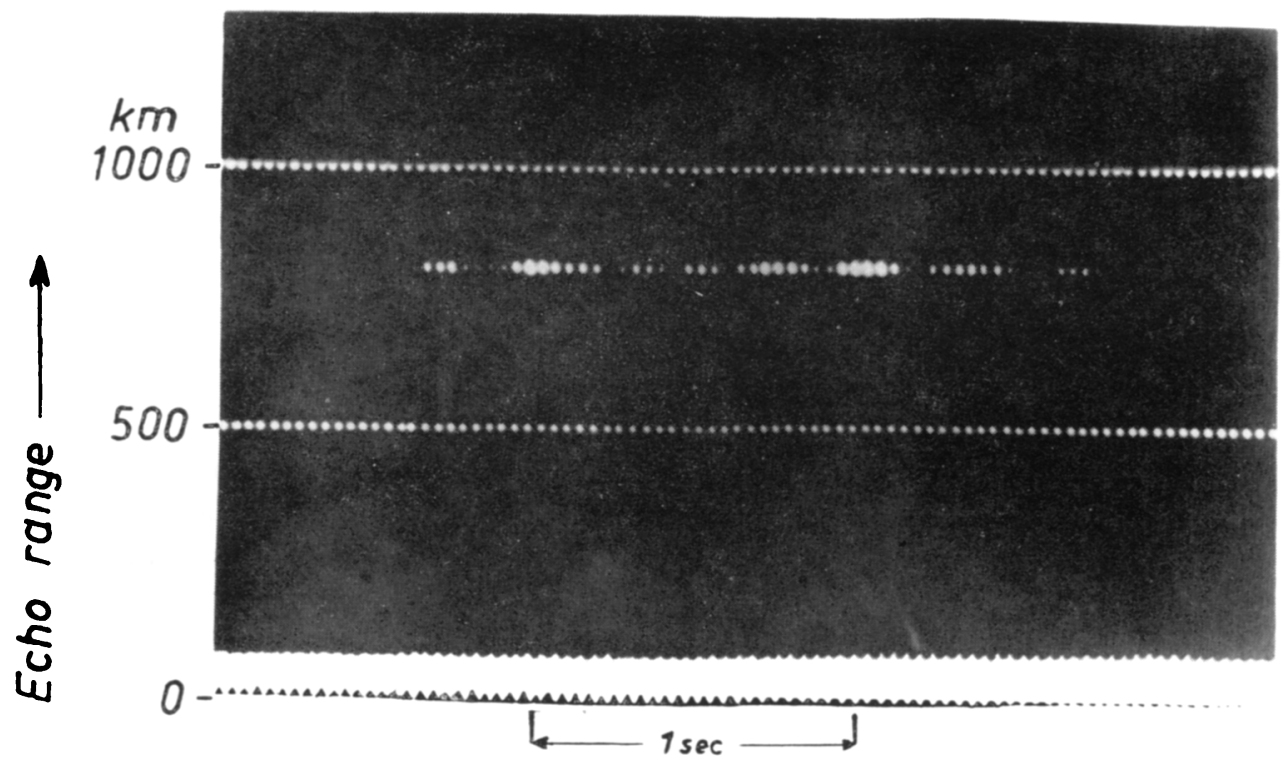

FIG. 1. Quadrantid meteor echo on the $33 \mathrm{Mc} / \mathrm{sec}$ radar, Kühlungsborn, January 2, 1967, 02:32 UT.

antenna the sensitivity of the radar was highest for meteors with radiants near the zenith. In special programs, the Quadrantid shower (January 3-4) was investigated since 1963, and the durations of 3000 Quadrantid meteors were measured. The high echo rates during the 2 or 3 days every year of Quadrantid activity made sure that the background of sporadic meteors was negligible, so that the recorded echoes could be attributed to meteors with a distinct geocentric velocity. The probability of occurrence of echoes with durations $T_{\mathrm{i}}$ exceeding $T$ is

$$
S(T)=\begin{gathered}
N\left(T_{\mathrm{i}} \geqslant T\right) \\
N\left(T_{\mathrm{i}} \geqslant 0.5 \mathrm{sec}\right)
\end{gathered} .
$$


$S(T)$ was calculated from the observational material separately for night- and daytime observations (Figure 2). Fortunately the time of radiant culmination of the Quadrantids (07:45 UT $=08: 34$ Local Time) is near sunrise, thus making it possible to observe Quadrantids during darkness as well as during day. The twilight time $\left(100^{\circ} \geqslant z_{\odot} \geqslant 90^{\circ}\right) *$ was excluded, in order to get a clear separation between night and day light conditions, bearing in mind that the infrared radiation reaching the meteor region even at negative sun elevations could cause severe detachment.

As Figure 2 indicates, there are significantly more long-duration meteors during

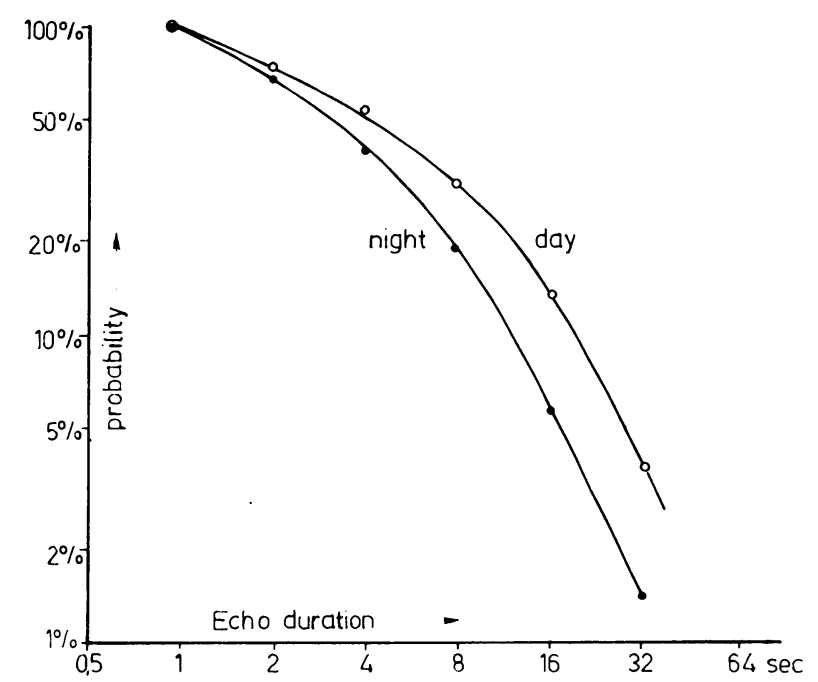

FIG. 2. Echo duration probability distribution of observed Quadrantids 1963-67, separated for day-and night-time.

day-time than during night. This can be due only to a process, which shortens the echo duration during night, but not during day-time. As calculated below, attachment is effective only during night, whereas the sunlight causes a high detachment rate.

A certain value of probability $S$ should refer to a certain mass or magnitude of meteors during day as during night. A change of mass distribution depending on the ecliptic longitude (Kaščeev and Lebedinec, 1961) would be compensated, because Figure 2 contains results of 5 years of observation, during which day and night meteors are divided at different ecliptic longitude.

The value $S=0 \cdot 1$, belongs during night to the duration $T_{A}=12 \mathrm{sec}$, but during day to the duration $T_{D}=19 \mathrm{sec}$. This means that a meteor echo which would last $19 \mathrm{sec}$ in day-time, is shortened by attachment to $12 \mathrm{sec}$ at night. From such pairs of equal probability taken from Figure 2 the effective attachment rate $A$ can be calculated.

$* z_{\odot}=$ solar zenith angle. 
The attachment causes an exponential decrease of line density with time. The electron density $n_{\mathrm{e}}$ then is given by

$$
\frac{\delta n_{\mathrm{e}}}{\delta t}=D \times \Delta n_{\mathrm{e}}-n_{\mathrm{e}} \times A+\left(n_{\mathrm{p}}-n_{\mathrm{e}}\right) \times \mathrm{C} .
$$

$D$ is the ambipolar diffusion coefficient, and the first term on the left describes the gaussian distribution of free electrons in the trail. Because the attachment, which is described by the second term, is proportional to $n_{\mathrm{e}}$, the distribution remains gaussian. The third term refers to the detachment, $n_{\mathrm{p}}$ is the number density of the positive ions or of the electrons in case of no attachment, which means

$$
\begin{gathered}
\delta n_{\mathrm{p}} \\
\delta t
\end{gathered}=D \times \Delta n_{\mathrm{p}} .
$$

$n_{\mathrm{p}}-n_{\mathrm{e}}$ is the number density of negative ions, $C$ is the detachment rate. The solution of (2) is given by

$$
n_{\mathrm{e}}=n_{\mathrm{p}}(r, t)\left\{e^{-(\boldsymbol{A}+C) t}+\frac{C}{A+C}\left(1-e^{-(A+C) t}\right)\right\} .
$$

The attachment causes an exponential decrease in electron density, which would be equal to $n_{\mathrm{p}}$ in case of diffusion only. The duration $T$ of an overdense echo is the time, during which in the centre of the trail $(r=0)$ the electron density $n_{\mathrm{e}}(r, T)$ exceeds the critical value. If $T_{0}$ is the echo duration in case of diffusion only, the echo duration with attachment becomes

$$
T_{A}=T_{0}\left\{e^{-(A+C) T_{A}}+\frac{C}{A+C}\left(1-e^{-(A+C) T_{A}}\right)\right\} .
$$

If detachment is negligible $(A \gg C)$, this yields possible values of $T_{A}$ :

$$
T_{A}=T_{0} e^{-A \times T_{A}} .
$$

$A$ depends on the height $h$ of the echo, or, as $h$ and the air density $\rho$ vary with the original meteor masses $m$, and $m$ can be expressed by the attachment-free echo duration $T_{0}, A$ depends on $T_{0}$. It is $\rho \sim m^{1 / 3}, m \sim T_{0}^{3 / 4}$ and therefore $\rho \sim T_{0}^{1 / 4}$, so that (5) may be written

$$
T_{0}=T_{A} e^{A\left(T_{0}\right) \times T_{A}} .
$$

In consequence of the uniform velocity of the Quadrantids the height of maximum ionization should depend on the mass of the meteor only, if the small echo height variations of less than $2 \mathrm{~km}$ because of the changing zenith distance of the radiant $\left(6^{\circ}<z<40^{\circ}\right)$ during the time of observation (03.45-11.45 UT) are neglected.

From (5a) and the experimentally found distributions of Figure 2, the attachment rate $A\left(T_{0}\right)$ can be calculated (Figure 3 ). The significance of this calculation is low for 


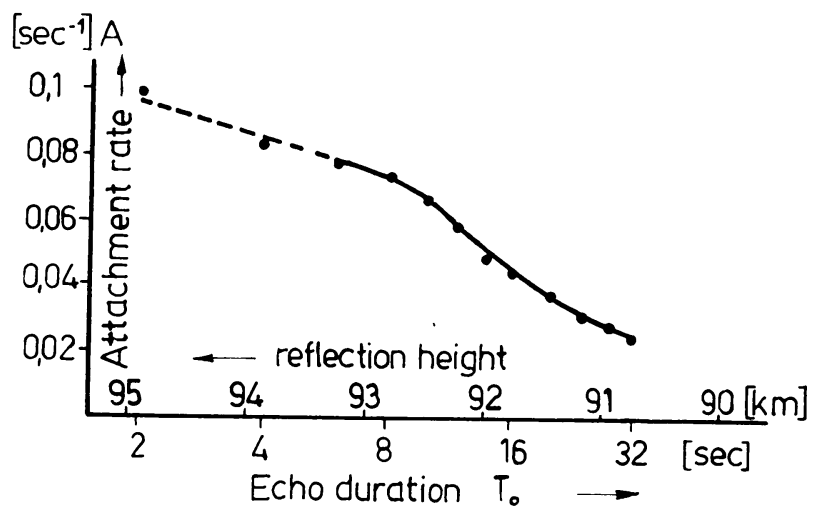

FIG. 3. Attachment rate of Quadrantids deduced from the day-night differences of Figure 2.

shorter echoes, because from relatively small differences in the distributions, high values of $A$ are deduced due to the factor $T_{A}$ in the exponent. On the other hand, very long enduring echoes are rare, and therefore their numbers are too small for a proper statistical analysis, so that these parts in Figure 3 are indicated by dashes only.

The Leonids, which in 1965 brought echo rates five times higher than in the years before, show day-night differences in the probability distribution of the same order as the Quadrantids (Figures 4 and 5). Due to the higher velocity of the Leonids $(v=$ $72 \mathrm{~km} / \mathrm{sec}$ ), the same echo duration for the Leonids refers to an air density smaller than for the Quadrantids $(v=42 \cdot 7 \mathrm{~km} / \mathrm{sec})$ by a factor $\left(v_{L} / v_{Q u}\right)^{-2}=0 \cdot 35$. This estimate is correct for an ionization factor $\beta \sim v^{2}$ in the relation $q_{\max } \sim m \times \beta(v)$ for the calculation of the echo duration $T \sim q_{\max } / D \sim q_{\max } \cdot \rho$. In case of $\beta \sim v^{3}$ the factor

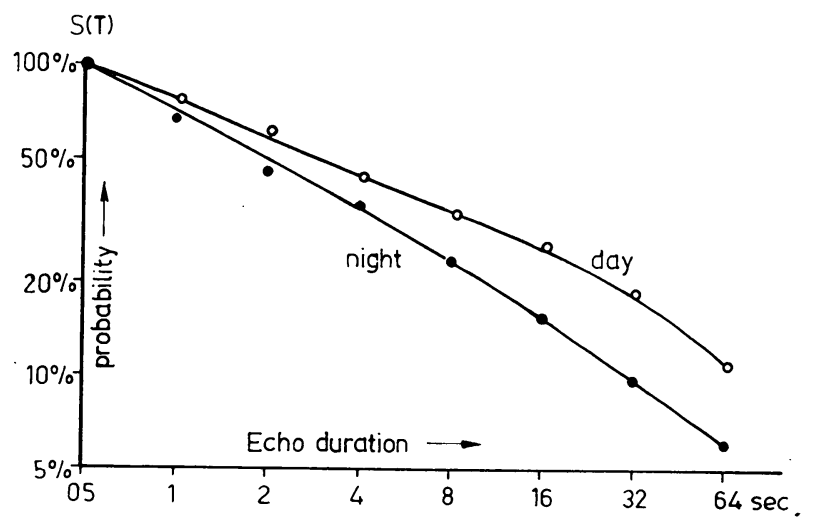

FIG. 4. Echo duration probability distribution of observed Leonids 1965, separated for day- and night-time. 


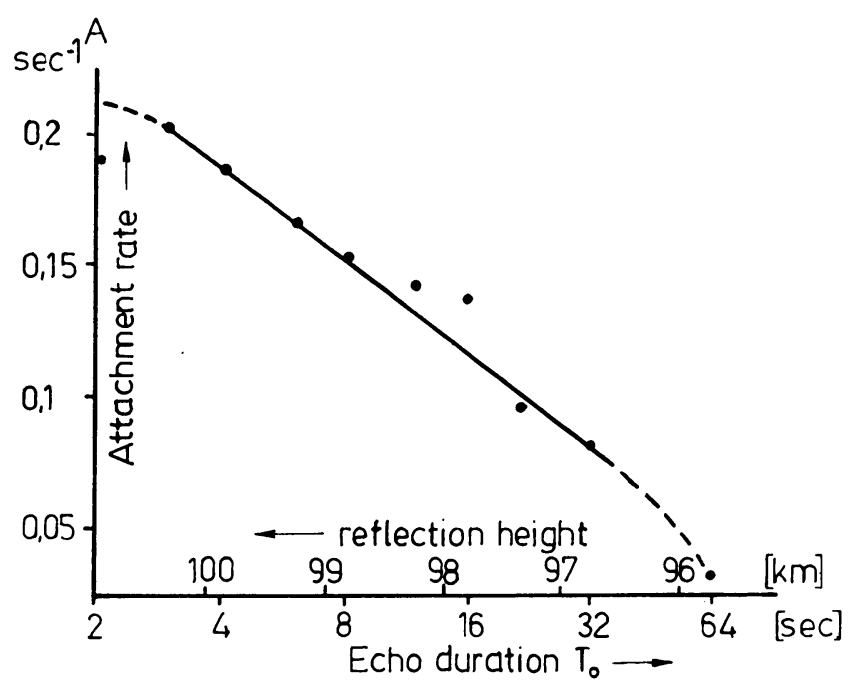

FIG. 5. Attachment rate of Leonids deduced from the day-night differences of Figure 4.

becomes $\left(v_{L} / v_{Q u}\right)^{-9 / 4}=0 \cdot 33$. In any case an echo of the same duration $T_{0}$ would come from a region about one scale height $(\approx 6 \mathrm{~km})$ higher for the Leonids than for the Quadrantids, or the same aeronomical conditions and therefore the same attachment rate would be proper for a Leonid with a duration of 66 times the duration of a Quadrantid. This means, that the same attachment rate should be expected for Quadrantids of $T_{0}=1 \mathrm{sec}$ duration as for Leonids of $T_{0}=66 \mathrm{sec}$ duration. Therefore Figure 5 fits well as the continuation of Figure 3, but the strong increase of attachment rates with echo height cannot be explained and is probably not real.

The results of Perseid observations in August 1967 are given in Figure 6. There are again significant day-night differences of echo durations, leading to attachment rates of $0.006-0.012 \mathrm{sec}^{-1}$ in heights of $97-94 \mathrm{~km}$. For the Perseids (August) the attachment rate increases with echo duration or decreases with meteor height and is generally smaller than deduced from the Quadrantid (January) and Leonid (November) observations, so that a seasonal variation could be suspected.

\section{Aeronomical Considerations}

The attachment of free electrons to neutral particles is an exothermal reaction, the generated energy of $1.5 \mathrm{eV}$ (attachment to atomic oxygen) or $0.46 \mathrm{eV}\left(\mathrm{O}_{2}\right)$ can either be radiated by a process

$$
X+e \rightarrow X^{-}+h v,
$$

which would be proportional to the density $n(X)$, or the energy can be taken by a 


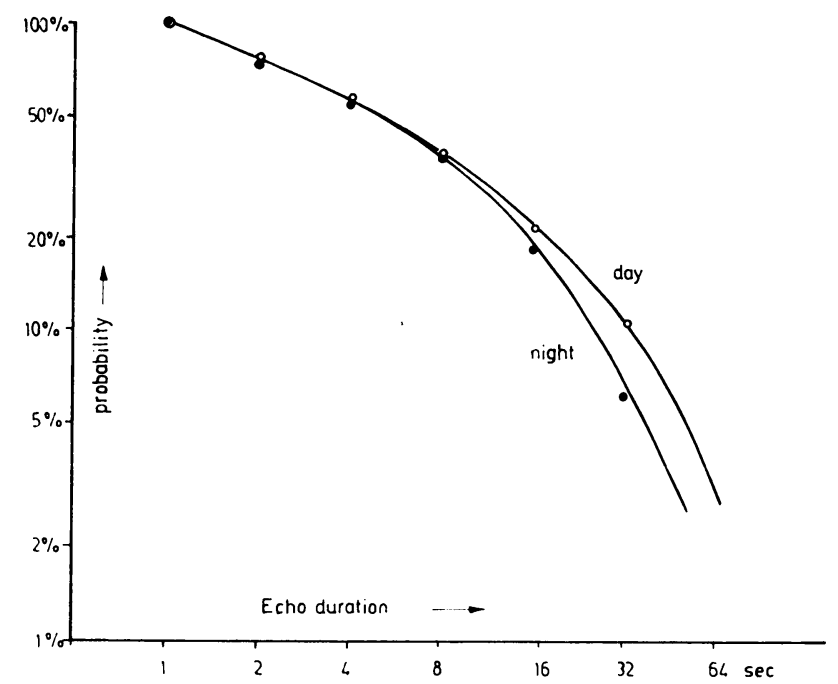

Fig. 6. Echo duration probability distribution of observed Perseids 1967, separated for day-and night-time.

third particle

$$
X+e+Y \rightarrow X^{-}+Y^{\prime}
$$

leading to attachment rates proportional to $n(X) \times n(Y)$. A third kind of process is the dissociative attachment like

$$
\mathrm{O}_{3}+e \rightarrow \mathrm{O}^{-}+\mathrm{O}_{2} \text {. }
$$

Table 1 contains the attachment processes of oxygen and their rate coefficients after Wagner (1964), together with the densities of the involved particles (COSPAR, 1965 ) and the calculated attachment rate $A$.

None of the mentioned reaction rates is high enough to explain the attachment rates found experimentally (Figure 3) or deduced by other authors (Manning, 1964b; Plavcová, 1965). The theory of Manning (1964a) assumes a height dependence of $A$ as $A_{0} \times \exp \left(m\left(h_{0}-h\right)\right) / H$ with $m=1$ for two-body attachment, $m=2$ for three-body attachment. In case of atomic oxygen, which has maximum concentration at $100 \mathrm{~km}$ height, the attachment rate $A$ will decrease with decreasing height ; between $95-105 \mathrm{~km}$ it will be nearly constant $(m=0)$, but below $100 \mathrm{~km}$ in the region of overdense meteors it never increases like the air density $\rho$ or like $\rho^{2}$.

Figure 3 probably indicates a decrease of $A$ with increasing duration or decreasing height, e.g. an attachment to $O$. But the concentration of $O$ decreases during night and should be even smaller than listed in Table 1 during the post-midnight observations. Since the rates of reaction $V$ of Table 1 are too small, the three-body attachment (reaction VI) may be important, despite the low concentration of $\mathrm{O}$, but 


\section{Table 1}

\section{Attachment processes of molecular and atomic oxygen and ozone}

reaction

I. $\mathrm{O}_{2}+e \rightarrow \mathrm{O}_{2}^{-}+h v$

II. $\mathrm{O}_{2}+e+\mathrm{O}_{2} \rightarrow \mathrm{O}_{2}^{-}+\mathrm{O}_{2}^{\prime}$

III. $\mathrm{O}_{2}+e+\mathrm{N}_{2} \rightarrow \mathrm{O}_{2}^{-}+\mathrm{N}^{\prime}$

IV. $\mathrm{O}_{2}+e \rightarrow \mathrm{O}^{-}+\mathrm{O}$

V. $\mathrm{O}+e \rightarrow \mathrm{O}^{-}+h v$

VI. $\mathrm{O}+e+\mathrm{M}^{\rightarrow} \mathrm{O}^{-}+\mathrm{M}^{\prime}$

VII. $\mathrm{O}_{3}+e \rightarrow \mathrm{O}^{-}+\mathrm{O}_{2}$ attachment rate constant

$85 \mathrm{~km}$

density

$90 \mathrm{~km}$

$95 \mathrm{~km}$

$85 \mathrm{~km}$

attachment rate

$90 \mathrm{~km}$

$95 \mathrm{~km}$

$\beta\left(\mathrm{O}_{2}\right)=2 \times 10^{-18} \mathrm{~cm}^{3} \mathrm{sec}^{-1} \quad n\left(\mathrm{O}_{2}\right)=3.25 \times 10^{13} \quad 1.33 \times 10^{13} \quad 5.2 \times 10^{12} \mathrm{~cm}^{-3} \quad A=6.5 \times 10^{-5}$ (Wagner, 1964)

$\omega\left(\mathrm{O}_{2}\right)=2 \times 10^{-30} \mathrm{~cm}^{6} \mathrm{sec}^{-1}\left\{n\left(\mathrm{O}_{2}\right)\right\}^{2}=1 \times 10^{27} \quad 1.8 \times 10^{26} \quad 2.7 \times 10^{25} \mathrm{~cm}^{-6}$ (Wagner, 1964)

$\omega\left(\mathrm{N}_{2}\right)=10^{-31}$, efficiency only $1 / 50$ of reaction (II), therefore meaningless

(Chanin et al., 1962; Van Lint and Wyatt, 1963) (Branscomb, 1964)

$\beta\left(\mathrm{O}_{3}\right)=4 \times 10^{-11} \mathrm{~cm}^{3} \mathrm{sec}^{-1} \quad n\left(\mathrm{O}_{3}\right)=2.5 \times 10^{7} \quad 1 \times 10^{7} \quad 3 \times 10^{6} \mathrm{~cm}^{-3}$ (Fehsenfeld et al., 1967)

$A=6.5 \times 10^{-5} \quad 2.7 \times 10^{-5} \quad 1 \times 10^{-5}$

$A=2 \times 10^{-3} \quad 3.6 \times 10^{-4} \quad 5.4 \times 10^{-5}$ (no special night-time data available) 
the rate coefficient is as yet unknown. The dissociative attachment to $\mathrm{O}_{3}$ has a high rate coefficient and is probably the dominating reaction, but the $\mathrm{O}_{3}$ concentration is not sufficiently known, especially its increase during night, which favours reaction VII.

Following the model of Hunt (1966) for the pre-dawn ionosphere the primary $\mathrm{O}^{-}$ formation will be the main free-electron sink above $65 \mathrm{~km}$ during night. The attachment to nitrogen and to minor constituents should be unimportant, the other negative ions are created by charge transfer, which finally stops at the ion $\mathrm{NO}_{2}^{-}$(Fehsenfeld et al., 1967). $\mathrm{NO}_{2}^{-}$was the only other negative ion found on a rocket-borne mass spectrometer (Johnson et al., 1958). But these charge-transfer processes are too slow to influence the meteor echo duration. The dissociative attachment to $\mathrm{O}_{2}$ (reaction IV) requires electrons of more than thermal energies; it is a resonance process for electrons of 6-7 eV.

The attachment is counterbalanced by detachment, which produces again free electrons from the negative ions $\mathrm{O}^{-}$or $\mathrm{O}_{2}^{-}$. The energy for the detachment is provided either by solar radiation, or by collision, or by collision and production of new molecules. According to Equations (2)-(4) the detachment rate $C$ is the sum of the rates of the radiation and collision detachment processes of the certain kind of negative ion (assuming that always a certain kind of negative ion dominates), that means $C=\kappa+\sum_{i} n_{\mathrm{i}} \times \zeta_{\mathrm{i}}$. Most problematic are the rate coefficients of collisional detachment with formation of new molecules, for which Fehsenfeld et al. (1967) found from laboratory experiments very high rate coefficients leading to detachment rates $C$ much higher than hitherto expected:

$$
\begin{array}{ll}
\mathrm{O}^{-}+\mathrm{O} \rightarrow \mathrm{O}_{2}+e ; & \zeta=1.9 \times 10^{-10} \mathrm{~cm}^{3} \mathrm{sec}^{-1} \\
C=24 \mathrm{~s}^{-1}(90 \mathrm{~km}) & C=16 \mathrm{~s}^{-1}(80 \mathrm{~km}) \\
\mathrm{O}_{2}^{-}+\mathrm{O} \rightarrow \mathrm{O}_{3}+e ; & \zeta=1.25 \times 10^{-11} \mathrm{~cm}^{3} \mathrm{sec}^{-1} \\
C=41 \mathrm{~s}^{-1}(90 \mathrm{~km}) & C=28 \mathrm{~s}^{-1}(80 \mathrm{~km}) .
\end{array}
$$

If these rates are accepted, attachment would be unimportant for meteor echo duration, because the attachment rates always would be much smaller than detachment for the negative ions of oxygen, which are considered the most probable primaries. In this case the attachment to the meteor matter itself has to be considered, if attachment is not to be rejected at all.

From other ionospheric processes smaller collisional detachment rates with a rate coefficient $\zeta=10^{-20}$ seem to be correct (Wagner, 1964), so that for $\mathrm{O}^{-}$and for $\mathrm{O}_{2}^{-}$ during night $A \gg C$.

The detachment by radiation,

$$
\mathrm{O}^{-}+h v \rightarrow \mathrm{O}+e
$$

requires energies of $h v \geqslant 1.5 \mathrm{eV}$ or radiation with $\lambda \leqslant 8260 \AA$. During day-time the 
detachment rate by solar radiation reaches, for $\mathrm{O}^{-}, \kappa\left(\mathrm{O}^{-}\right)=1.4 \mathrm{sec}^{-1} \gg A$, so that practically all attachment processes to atomic oxygen are reversed immediately and therefore become inefficient. In Equation (3) $C \gg A$ and therefore $n_{\mathrm{e}} \approx n_{\mathrm{p}}$. Even assuming very high attachment rates $(A=0.07)$, Equation (3) gives $T_{A}=$ $T_{0}\left\{0.95+0.05 e^{-1.47 T_{A}}\right\}$, indicating that during day-time attachment can reduce the echo duration only by $5 \%$ at the most. As for $\mathrm{O}^{-}$the radiation detachment for $\mathrm{O}_{2}^{-}$is severe during day-time with $\kappa\left(\mathrm{O}_{2}^{-}\right)=0.44 \mathrm{sec}^{-1}$, that means $C \gg A$, so that during day-time practically no attachment remains.

\section{Conclusions}

(1) The differences of echo durations as seen from the probability distributions of night- and day-time observations of the Quadrantid, Perseid and Leonid showers indicate a strong influence of attachment during night and give a possibility for quantitative estimation of attachment rates.

(2) This estimate is based upon the aeronomical deduction, that in the sunlit atmosphere the detachment dominates all attachment processes and therefore no electrons remain attached.

(3) The rate coefficients and particle densities of the attachment processes are only partially known. The attachment rates of processes so far known cannot explain the high attachment rate, which results from the difference of night- and day-time probability distributions of echo duration or from previous investigations of other authors.

(4) The dissociative attachment to Ozone $\left(\mathrm{O}_{3}+e \rightarrow \mathrm{O}^{-}+\mathrm{O}_{2}\right)$ has a high rate coefficient $\beta\left(\mathrm{O}_{3}\right)=4 \times 10^{-11} \mathrm{~cm}^{3} \mathrm{sec}^{-1}$, so that pre-dawn Ozone concentrations of $n\left(\mathrm{O}_{3}\right) \approx 10^{9}$ particles per $\mathrm{cm}^{3}$ could explain the attachment rate. Otherwise attachment to $\mathrm{O}$ would be important above $90 \mathrm{~km}$, whereas at $90 \mathrm{~km}$ and below the rates of three-body attachment to $\mathrm{O}_{2}$ would dominate.

(5) The general decision between two-body or three-body attachment (as proposed by Manning) seems impossible, if attachment to atomic oxygen is involved, because the density of $\mathrm{O}$ has its maximum at $100 \mathrm{~km}$ height and decreases therefore with decreasing echo height, and cannot be expressed by $n(0) \sim T^{1 / 4}$ (two-body attachment) or $\{n(\mathrm{O})\}^{2} \sim T^{1 / 2}$ (three-body attachment).

(6) If the detachment rates reported by Fehsenfeld et al. (1967) are valid in the ionosphere, collisional detachment would dominate during both day and night and would break off any attachment to oxygen. Thus either attachment is completely unimportant, or attachment to meteor matter must be considered.

\section{References}

Branscomb, L. M. (1964) Ann. Géophys., 20, 88.

Chanin, L. M., Phelps, A. V., Biondi, M.A. (1962) Phys. Rev., 128, 219. 
COSPAR (1965) Int. Ref. Atmosph., North-Holland Publ. Co., Amsterdam. Davis, J., Greenhow, J.S., Hall, J.E. (1959) Proc. Roy. Soc., A 253, 130.

Fehsenfeld, F.C., Schmeltekopf, A.L., Schiff, H.I., Ferguson, E.E. (1967) Planet. Space Sci., 15, 373.

Greenhow, J.S., Hall, J.E. (1962) J. atmos. terr. Phys., 21, 261.

Hunt, B.G. (1966) J. geophys. Res., 71, 1385.

Johnson, C. Y., Meadows, E. B., Holmes, J.C. (1958) J. geophys. Res., 63, 443.

Kaiser, T.R. (1953) Adv. Phys., 2, 495.

Kaščeev, B.L., Lebedinec, V.N. (1961) Rezultaty Issled. MGG - Ionosfera i Meteory, No. 7, 1. Manning, L.A. (1964a) Radio Sci. J. Res. NBS/USNC-URSI, 68D, 1067.

Manning, L.A. (1964b) Radio Sci. J. Res. NBS/USNC-URSI, 68D, 1079.

McKinley, D. W. R. (1961) Meteor Science and Engineering, McGraw-Hill Book Co., New York. Plavcová, Z. (1965) Bull. astr. Inst. Csl., 16, 127.

Van Lint, V.A.J., Wyatt, M.E. (1963) 16th Annual Gaseous Electronics Conference, Pittsburg. Wagner, C.U. (1964) Vorträge der Sommerschule Untere Ionosphäre, NKGG-Veröff., Berlin, 2,127 .

\section{DISCUSSION}

Kaiser: I wish to make two observations. First, dual wavelength radar observations and data from the decay of luminous meteor trains give similar values for the attachment coefficient and indicate a three-body process. Secondly, we have observed a dramatic sunrise effect during Quadrantid observations, namely a sudden increase in the number of non-specular enduring echoes. The time of occurrence of this effect coincides with the solar grazing of the ozonosphere.

Glöde: Still the rates for three-body attachment processes deduced from aeronomical considerations are smaller than those found for meteor echoes.

McIntosh: With regard to Leonid observations I make three comments:

(1) During the strong return in 1965 there was a real change in the mass distribution throughout the period of the shower. The Ottawa results are supported in this by the Czechoslovak results (Plavcová, in the present volume p. 432.).

(2) We find that attachment does not affect Leonid echo duration until $T>32 \mathrm{sec}$, the value where Dr. Glöde's results terminate.

(3) I find that a best fit to Leonid duration is obtained for an attachment rate of $0.02 \mathrm{sec}^{-1}$ at $93 \mathrm{~km}$.

Glöde: An effect of change in mass distribution in the case of Leonids cannot be completely excluded, but we put together the meteors observed on 4 days and nights around the date of maximum, so that this effect should not cause the significant day-night differences.

Certainly the attachment becomes more remarkable for longer enduring echoes. The estimated attachment rate for the Leonids seems indeed too high, so that your value of 0.02 seems to me the better one. For quantitative measurements the number of Leonid meteors observed at Kühlungsborn probably was too small.

Elford: What were the actual periods during which the night-time and day-time observations were carried out? Were they just before and after sunrise?

Glöde: We excluded the time, when the solar zenith angle $z \odot$ was $100^{\circ}>z \odot>90^{\circ}$. For the statistical analysis we used mostly the two hours before and after that time. To avoid influences of changing zenith distance of the radiant only measurements at zenith distances smaller than $40^{\circ}$ were taken into account.

Millman: What percentage of non-Quadrantid meteors do you estimate in your total of 3000 observed echoes?

Glöde: Due to the horizontally beamed antenna an upper limit of $10 \%$ sporadic meteors may have entered the analysis.

Bronšten: We can also take into consideration the reactions

$$
\begin{aligned}
\mathrm{N}_{2}+\mathrm{O}_{2} & \rightarrow \mathrm{NO}^{+}+\mathrm{NO}^{-} \\
\mathrm{NO}^{+}+e & \rightarrow \mathrm{N}\left({ }^{2} D\right)+\mathrm{O} .
\end{aligned}
$$


At the height of $100 \mathrm{~km}$ the ratio $\left(\mathrm{N} / \mathrm{N}_{2}\right)$ is lower than $10^{-3}$, but it is due to the reaction

$$
\mathrm{NO}+\mathrm{N} \rightarrow \mathrm{N}_{2}+\mathrm{O} \text {. }
$$

Glöde: The first reaction does not remove electrons, it could enhance the number of free electrons by detachment from $\mathrm{NO}^{-}$. The other reaction is a recombination process which is thought to be of lower efficiency than attachment due to the small density of $\mathrm{NO}^{+}$. The third reaction contributes to the density of atomic oxygen.

Kaiser: We should remember that atmospheric motions can affect the duration of radar echoes, thus a change at sunrise in these motions could contribute to a day-night difference in echo duration. Although I believe the evidence is strongly in favour of attachment as the primary process, we should nevertheless examine meteor wind data in order to estimate any effect of a change in the nature of air motions between night and day.

Glöde: Especially if attachment as the main cause of electron loss during night should be ruled out by high detachment rates as mentioned above, atmospheric turbulence has to be considered as a trail-disturbing process, which could cause strong day-night differences. With sunrise, turbulence should increase and shorten the echo duration, but also shift new trails into specular condition, which could increase the ratc of long-enduring echoes. 